(Aus der paychiatrisehen Klinik der Stoekholmer Herenheilanstalt

IChef: Prof. Dr. Ciadoliusil.)

\title{
Blutuntersuchungen bei einem Fall von Dementia praecox mit periodischem Verlauf.
}

\author{
Von \\ Gunnar Kahlmeter, \\ Irüh. Assistenzarzt an der Stockholmer Irrenheibunstalt.
}

Mit 2 Textfiguren.

(Eingegangen am 2s. Februar 1914.)

X. E. W., geb, 1876, vorheiratet, 4 Kinder.

Eltern nicht verwandt; weder Vater noch Muttes haben Eigentümlichkeite'n im Charakter, Benehmen oder Lebensgewohnheiten aufgewiesen. Keine Fälle von Nerven-oder Geisteskrankheiten sind in der Familie bekannt, ausgenommen, dal3 II.'s Großnutter mütterlicherseits, die im Alter von 81 Jahren von einem Schlaganfall nit hochgradiger Lähmung betroffen wurde, danach ,geistesschwach" wirde.

IV. ist hervorragend begabt gewesen; er hat recht ungewöhnliche mathematische und praktische Anlagen gezeigt und ist auch sehr musikalisch begabt gewesen. Sein Wesen soll stets verschlossen und selhstkritjsch gewesen sein. Er ist verheiratet und hat + Kinder, alle gesund. Seine ökonomische Stellung ist gut, 'r hat unter glücklichen Familienverhältniwsen gelebt und eine geordnete Lebensweise geführt, keinen AlkoholmiBbrauch getrieben, ist sich luetiseher Infektion nicht bewußt.

W., der sich stets zuvor der besten (iesundheit erfreut hatte, begann im Herbst 1909, ,nervös, abwesend und merkwïrdig " zu werden, doch können diese Symptome nicht sehr auffallend gewesen sein, da scine Frau erst später bei näherem Nach. denken sich derselben (rimnerts. W., der Direktor einer großen Aktiengesellsehaft war, hatte whihrend jener Zeit wegen sinkender Konjunkturen wahrseheinlieh gewehätliche Sorgen und mußte cinige voll seinem l'crsonal entlassen, was ihn tief schmerzte. Während des Herbstes war er mit der Vertretung einer Professur beauftragt, um die e' sich auch \% bewerben gedachte. Während der Ausarbeitung riner Abhandlung, die dieser Bewerbung dienen sollte, mußte or seine Arbeit in hohem (irade forrieren, jede Nacht $l$ is gegen 3 oder 4 Uhr morgens durcharbeiten. Dor Gedanke an das Schicksal seiner Bewerbung um die Professur bereitete ihm auch viel Unruhe.

Nachdem er während der letzten Zeit an schwerer Schlaflosigkeit gelitten hatte, brgann WI. am 9. X. 1909 e cinige cigentümliche Äußerungen darüber zu fällen, daß "r glaubte, einige seiner Freunde intrigierten gegen seine Bewerbung um die Professur. Die Nacht darauf schlief er sehr sehlecht, sprang mitten in der Nacht aus dem Bett auf und lief die 'Treppo hinab, um einen Freund zu suchen, von dem er glaubte, daß er im Hause vorhanden se. Am 'Tage darauf (10. X.) lag er meistens ruhig, indem cr unaufhörlich die Xamen seiner Freunde wiederholte. Er erkannte 
seine Angehörigen und war zeitweise völlig klar; einigemal rief er aus: „Nun versteh' ich, weshalb ich wahnsinnig geworden bin." In der Nacht auf den 11. wurde er mehr und mehr verwirrt und so gewalttätig, daß zwei Männer nötig waren, ihn im Bett zu halten. Den Stuhl ließ er ins Bett gehn.

Am 11. X. wurde W. in eine Privatheilanstalt verbracht. Somatisch war nun nichts Abnormes zu bemerken außer Fieber. Wassermanns Reaktion im Blute war negativ. Patient war vollkommen verwirt, äußerst agitiert, warf sich im Bett oder in der Badewanne hin und her, schlug die Hände oder den Kopf heftig gegen die Wände, leistete beim Füttern intensiven Widerstand, ließ Harn und Stuhl ins Bett gehen. Die Stimmung war meistens aufgeräumt, bisweilen jedoch düster und ängstlich. Auf Anreden antwortete er in der Regel nicht, er lag bisweilen ganz still, meistens aber sang und schwatzte er vor sich hin. Hier und da konnte ein Satz vollständig sein, gewöhnlich aber war die Rede ganz verwirrt, sinnlos, aus zusammenhanglosen oder aus verdrehten, neugebildeten Wörtern bestehend. Oft war eine Neigung zu reimen bemerkbar, und oft wiederholte er Fragen oder Äußerungen der Umgebung. Fr halluzinierte sichtlich ziemlich viel, schlief trot\% Schlafmittel schlecht.

Während der nächsten Wochen wurde $W$. etwas ruhiger, zeitweise war (' sogar einigermaßen klar und orientiert. Gegen Ende des Jahres $1909 \mathrm{kam}$ er in einen Zustand, in welchem Tage, an denen er völlig klar und orientiert war, abwechselten mit Tagen, an denen er ungefähr dasselbe Bild wie während der ersten Zeit darbot. Die verschiedenen Zustände waren von sehr wechselnder Dauer und die Übergänge sehr rasch. In seinem besseren Zustand war er vollständig klar, geordnet und ruhig, wohlorientiert, sprach zusammenhängend und klar, benahm sich in allen Hinsichten tadellos, während er aber geordnet und klar sprach, konnten plötzlich einige ganz sinnlose Worte kommen, er begann abwesend auszusehen, den Körper anzuspannen und eine Menge gespannter Bewegungen und Grimassen auszuführen, den Körper langsam nach rückwärts oder nach den Seiten hin zu bewegen, die Arme auszustrecken, die Hände bis zur außersten Girenze zu pronicren oder zu supinieren, die Finger zu beugen, zu strecken und mit ihnen zu zucken, die Augen zusammenzukneifen, die Stim zu runzeln, den Mund zu spitzen usw. Während dieser gespannten Bewegungen konnte er plötzlich aufspringen, die Betten nchmen und auf den Fußboden werfen, unter das Bett kriechen, einen Stuhl wegschieben, mit der Hand gegen die Wand schlagen u. dgl. Hierbei pflegte er unaufhörlich dieselben sinnlosen Wörter zu wiederholen, z. B. surba, surba, surba. Ein andermal konnte W. lange Zeit auf demselben Fleck ganz unbeweglich und abwesend stehen, wobei er den Speichel in großen Mengen sich im Munde ansammeln ließ. Ein paarmal sprang er ganz unvermutet aus scinem Bett auf und zer schlug eine Fensterscheibe, worauf er sich wieder hinlegte.

Ebenso rasch war im allgemeinen der Übergang zu dem geordneten und klaren Zustand. W. konnte dann nie eine Erklärung für scin Auftreten während der unruhigen Perioden geben, sagte jedoch bei einer Gelegenheit, daß er einen Zwang zu fühlen pflegte, die eigentümlichen Bewegungen mit Armen und Beinen auszuführen.

Bei verschiedenen Gelegenheiten sprach W. davon, da 3 er eigentümliche Tiere, farbige Flecke auf dem Laken usw. sähe. Er hatte auch Gehörshalluzinationen von Lesten, die aus der Ferne schwatzten, Dynamitschüssen, die abgefeuert wurden $\mathrm{u}$. a. m. Hypochondrische Wahnvorstellungen kamen oft vor, er erklärte blind zu sein, eine Schlange habe ihm beide Augen ausgestochen; er hatte das Gefühl, daß er alles Blut verloren habe, daß seine Genitalien veründert seien.

Im Frühling 1910 wurde der Zustand allmählich so gut, daß W. im Juni dio Heilanstalt verlassen konnte. Er war dann zu Hause bis Februar 1911. Während 
dieser Zeit hatte er zwar mehrere Unruheperioden von demselben Typus wie zuvor, sie waren aber nicht so schwer, und dazwischen konnte er sogar teilweise seiner alten Arbeit nachgehen. Anfang Februar $1911 \mathrm{kam}$ indessen ein Rückfall, der seine Wiederaufnahme in eine private Heilanstalt notwendig machte. Er war nun agressiv und gewalttätig und zeigte Spaltungssymptome und Sterotypien wie vorher. Im Juli reiste er nach München, wo er in Krae peli ns Klinik eine Woche lang zur Beobachtung aufgenommen wurde; er kam mit der Diagnose Dementia praecox heim.

Am 13. IX. wurde er in die Stockholmer Irrenanstalt übergeführt, wo er das ganze folgende Jahr über denselben unregelmäßigen Wechsel zwischen ruhigen und klaren Zeiten und Zeiten heftiger Unruhe mit allen oben beschriebenen Symptomen aufwies. Die Übergänge geschahen oft unglaublich rasch. Während er z. B. mit Tennisspiel beschäftigt war und vollständig gesund erschien, konnte er plötzlich das Racket hinwerfen, worauf er dann vor sich hin zu stieren, Grimassen zu schneiden, den Körper in die gewöhnlichen gespannten, stereotypen Stellungen zu verdrehen und zu rufen begann: „Nun kommt es wieder, nun werde ich wieder verrückt." Er konnte dann oft nur mit Hilfe einiger Wärter zu seinem Bett in der Abteilung der Unruhigen geführt werden. Ebenso rasch vollzog sich auch der Übergang in den ruhigen Zustand.

Gegen Ende 1912 begannen die Wechsel in W.'s Krankheit den Verlauf anzunehmen, den sie während der ganzen ersten Hälfte des Jahres 1913 behielten, und den sie also in der Zeit 11. III. bis 31. IV. hatten, während welcher ich regelmäßige Blutuntersuchungen an ihm machte. Während dieser Zeit zeigte W. einen sehr regelmäßigen Wechsel zwischen Zeiten, wo er vollständig symptomenfrei erschien, und Unruheperioden oben beschriebenen Charakters. Die freien Intervalle dauerten 7--8 Tage, die Unruheperioden ungefähr 5 Tage. Während der ersteren war $W$. völlig klar und geordnet, beschäftigte sich mit Slöjd, mit Billardund Lawntennisspiel, besonders aber mit Klavierspielen, worin er sehr geschickt war. Er pflegto während dieser Zeiten Besuche bei seiner Familie, die in der Nähe von Stockholm wohnte, oder auch bei Bekannten in der Stadt abzustatten, wobei er sogar Gesellschaften mitmachte. Sein Benehmen war hierbei völlig geordnet und unverworren. Die ersten Zeichen des Überganges in eine Unruheperiode waren stets eine leiche Hemmung oder 1)epression, begleitet von Starrheit und Stereotypie der Bewegungen. W. konnte dann lange Zeit dastehen und die Hand des Arztes halten, indem er abgebrochene Worte vor sich murmelte, wie z. B.: „Ich weiß nicht. ..", wobei Lippen und Cesichtsmuskeln sich in grimassenartiger Weise bewegten. Nach einigen Stunden wurde er plötzlich lärmend und stürmisch, war schwer im Bett zu halten, warf sich im Bett hin und her, sprang auf und nieder, lief im Zimmer umher, schwatzte, sang und schrie, scherzte, machte Witze und zeigte ein ziemlich offenkundige Ideenflucht. Er erkannte seine Umgebung, nannte aber alle „Du“. Seine Aufmerksamkeit konnte man nur für einen Augenblick fesseln, dann aber zeigte er sich zeitlich und räumlich ziemlich gat orientiert.

Nach 2-3 Tagen wurde er mehr starr und deprimiert, saß dann im allgemeinen im Bett, die Decke über den Kopf gezogen, laut weinend und klagend, sämtliche Muskeln gespannt und bretthart, dieHände fest zusammengeballt, oft in phantastische Stellungen verdreht und die oben beschriebenen athetosenartigen Bewegungen mit den Fingern ausführend. Die einzigen Worte, die W. zwischen dem Geschluchze hervorpreßte, pflegten dann zu sein: „Ich habe meine Frau gemordet, ich bin tot, ich habe dich gemordet" usw. Nicht selten ließ er den Harn, oder auch sogar die Exkremente, ins Bett gehen.

Nachdem diese Depressionsperiode ungefähr zwei Tage gedauert hatte, wurde er im Laufe einiger Stunden wieder völlig ruhig, geordnet und klar, und nachdem 
er noch vor einigen Stunden wie ein Knäuel zusammengerollt, steif und gespannt und laut weinend im Bett gelegen hatte, konnte er dann dasitzen und Beethoven spielen oder sich auf dem Wege zu seiner Familie befinden.

In den Zwischenzeiten fühlte er sich selbst völlig gesund und wie frühcr, ausgenommen, daß er ein Ciefühl davon hatte, daß er nicht wie früher würde arbeiten können, auch empfand er nicht mehr das gleiche Interesse wie früher für das, was ihn damals interessierte. Daß seine Interessen nun abgestumpfter und banale $\mathrm{Y}$ waren, als wie man es von einer Person, seiner Begabung und seines Bildungs. grades erwartet, war völlig klar.

W.'s somatischer Status war folgender. Länge $175 \mathrm{~cm}$, Gewicht $72 \mathrm{~kg}$. Keinc somatischen Degenerationszeichen. Herz, Lungen und Nieren normal. Thyreoidea nicht palpabel. Die Temperatur während der „freien Intcrvalle“ afebril, während dor Unruheperioden, besonders in den ersten Tagen, meistens etwas gesteigert, jedoch nis über $38^{\circ}$. Pupillen rund, gleichgroß, mittelweit, reagieren gut auf Licht und in bezug auf Akkomodation. Auch die übrigen Kranialnerven zeigen nichts Besonderes. Motilität und Sensibilität normal. Haut- und Sehnenreflexe normal.

Die Krankengeschichte betrifft also einen bei der Erkrankung 33 jährigen Mann, der in der Jugend sich hochbegabt, aber verschlossen und selbstkritisch gezeigt hatte. Nach einer Zeit forcierter, aufregender Arbeit, während der er sich etwas .,nervös, abwesend und merkwürdigc gezeigt hatte, erkrankt er im Oktoher 1909 plötzlich an einem Verwirrungszustand mit hochgradiger motorischer und psychischer Unruhe. Ziemlich bald wird er etwas ruhigęr und weist danach während des nächstfolgenden Jahres sehr wechselnde Zustïnde auf. An einigen Tagen ist er völlig klar, geordnet und besonnen, besitzt sogar Krankheitscinsicht, an anderen Tagen ist er verwirrt und gewalttïtig, zeigt eine Menge eigentümlicher stereotyper Bewegungen, impulsive, unmotivierte und zwecklose Handlungen, hypochondrische Wahnvorstellungen uml auch Halluzinationen.

Nachdem er während des Winters 1910-1911 eine längere ruhige Periode gehabt hat, bekommt er im Sommer 1911 einen Rückfall. Dils Krankheitsbild ist ungefähr dasselbe mit seinem scharfen Wechsel von heftiger Unruhe und scheinbarer Gesundheit mit unglaublich raschen Ubergängen zwischen den verschiedenen Zuständen. Gegen Ende des Jahres 1912 beginnt die Krankheit einen gewissen periodisehen Typu anzunehmen, indem die Unruheperioden ziemlich regelmäßig 5 Tage, die freien Intervalle $7-8$ Tage dauern. Dieser periodische Verlauf fährt unverändert fort, als ich im März und April 1913 Blutuntersuchungen an dem Patienten vornehme.

Die Diagnose in diesem Falle erschien anfangs sehr einfach. Die ganze Erkrankung mit der heftigen, unbeträchtlich affektiv gefärbten Unruhe der Menge von Stereotypien in Bewegungen und Rede, die bizarren, zwecklosen, impulsiven Handlungen, ausgeführt ohne augenfïlligen Affekt, alles wies auf Dementia praecox, und zwar zunächst auf Katatonie hin. Krapelin selbst, in dessen Kilinik der Patient cine 
Woche lang weilte, faßte gleichfalls den Fall als Dementia praecox auf.

Indessen hat der weitere Verlauf sich so gestaltet, daß man wohl doch nicht die Möglichkeit ausschließen kann, daß der Fall der manodepressiven Gruppe angehört. Was für diese Möglichkeit spricht, ist teils der Umstand, daß die Unruheanfälle in letzterer Zeit eine stärkere Affektfärbung zeigen (während der ersten Tage der Unruheperioden ist die Stimmung stets sehr gehoben, während des Höhepunktes der Periode deprimiert und während der letzten Tage wieder gehoben), teils der Umstand, daß trotz der mehrjährigen Dauer der Krankheit keine Demenz eingetreten ist. Der periodische Verlauf gewährt an und für sich keinen Anhaltspunkt für die Diagnose, er kommt innerhalb beider Krankheitsgruppen vor.

Jedoch weist $W$. während der Unruheperioden andauernd in so hohem Grade Stereotypien und Spaltungssymptome auf, daß ich, besonders mit Rücksicht auf die vorhergehende Krankengeschichte, immer noch es für das wahrseheinlichste halte, daß der Fall als Dementia praecox zu rubrizieren ist, möchte aber aus den oben angetührten Gründen die Möglichkeit einer manisch-rlepressiven Psychose offen halten.

An diesem Patienten nahm ich während längerer Zeit (11. III. his 30. IV. tägliche Blutuntersuchungen vor.

Prüft man die Untersuchungen über die Zusammensetzung des Blutes bei Geisteskranken, die in der Literatur vorliegen, so erweisen sich ihre Resultate als ziemlich unbestimmt und wenig miteinander übereinstimmend. Während einige Autoren keinerlei Abweichung von den normalen Verhältnissen gefunden haben, berichten andere von höchst beträchtlichen Veränderungen, die sowohl die Gesamtzahl roter und weißer Blutkörperehen als auch das prozentische Verhältnis zwischen den versehiedenen Arten weißer Blutkörperchen betreffen.

Die versehiedenen Autoren sind sich indessen keineswegs rlarüber einig, welcher Art diese Veränderungen sind. Die Ursache dieser bedauerlichen Tatsache ist wohl zum Teil in der sehr verschiedenartigen Technik sowohl bei der Entnahme der Blutprobe als bei der Ausführung der Blutkörperuntersuchungen zu suchen, die von verschiedenen Autoren in verschiedenen Ländern angewandt worden ist. Abweichungen in der Technik sind indessen etwas, was bei Blutuntersuchungen niemals ganz vermieden werden kann. Alle Untersuchungen, die an Blutproben ausgeführt worden sind, welche bei einer einzigen oder bei wenigen Gelegenheiten von einem und demselben Patienten entnommen wurden, sind daher schon aus diesem Grunde von recht unbedeutendem Werte, und die weitaus überwiegende Mehrzahl veröffentlichter Untersuchungen ist auf rliese Weise ausgeführt worden. Dazu kommt, daß verschiedene Autoren natürlich nicht ihre Patienten unter völlig gleichartigen Verhältnissen 
untersucht haben. Damit man nämlich Schlüsse aus derartigen Untersuchungen ziehen könnte, wäre natürlich, außer gleichartiger Technik bei allen Untersuchern, erforderlich, daß das Blut des gesunden Menschen eine im großen und ganzen bei allen Individuen und unter allen Verhältnissen konstante Zusammensetzung zeigte. Wir wissen jedoch, daß dieses letztere keineswegs der Fall ist. Nicht genug damit, daß die $\mathrm{Zu}$ sammensetzung des Blutes bei verschiedenen gesunden Personen ziemlich bedeutende Verschiedenheiten aufweisen kann, es variiert auch bei demselben Individuum unter verschiedenen Verhältnissen. Ich brauche nur an den Einfluß zu erinnern, den die Verdauung, Muskelarbeit, Menstruation usw. auf die Zusammensetzung des Blutes ausübt.

Aus dem Obigen geht hervor, wie wenig vergleichbar miteinander die Ziffern sein müssen, die verschiedene Autoren bei vereinzelten Untersuchungen des Blutes Geisteskranker erhalten haben, wenn sie auch noch so viele Individuen untersucht haben. Ein größerer Wert muß natürlich der Untersuchung des Blutes eines und desselben Individuums tagtäglich während einer längeren Zeit zukommen, wobei man sozusagen das Blutbild des Patienten bei verschiedenen psychischen Zuständen vergleicht. Ergibt sich bei einem solchen Verfahren ein gesetzmäßiger Typus des Blutbildes, so muß diesem Resultat weit größere Bedeutung beigemessen werden als den vereinzelten Blutuntersuchungen, da die Fehlerquelle, die in der mangelhaften Exaktheit der Technik liegt, hier keine Bedeutung besitzt (vorausgesetzt natürlich, daß der Untersucher stets dieselbe Technik anwendet).

Bevor ich zu dem Bericht über meine Blutuntersuchungen in diesem Fall übergehe, will ich in größter Kürze über die wichtigsten der Untersuchungen an Blut von an Dementia praecox leidenden Geisteskranken berichten, die bisher in der Literatur veröffentlicht worden sind.

Was zunächst die Autoren betrifft, die keine Serienuntersuchungen ausgeführt haben, so haben die meisten derselben, wie Purdrum und Wells, Kuhn, Itten, Heilemann, Krueger, Pförtner, eine normale oder mäßig vermehrte Gesamtzahl weißer Blutkörperchen, mit einer relativen Lymphocytose, d.h. prozentische Vermehrung der Anzahl der Lymphocyten gefunden.

Sandri fand eine relative Lymphocytose bei Katatonie, relative neutrophile Polynucleose bei Hebephrenie, und Rougeau, der selbst 53 Fälle untersucht und 122 Fälle aus der Literatur, hauptsächlich der französischen und italienischen, gesammelt hat, fand meistens eine polynucleäre Leukocytose, besonders bei Patienten mit Halluzinationen. Es ist jedoch unmöglich zu entscheiden, wie viele von seinen Fällen der Dementia praecox zugehören.

Dide, der teils zusammen mit Chenois 18 Fälle von Dementia 
praecox untersucht, teils die Literatur daraufhin geprüft hat, falst seine Befunde folgendermaßen zusammen:

1. Die Hypoglobulie ist bei Geisteskranken der Regel nach Zeichen einer Intoxikation oder Infektion.

2. Die Hyperglobulie ist Zeichen einer vermehrten Konzentration des Blutes und findet sich daher bei Unruhezuständen.

3. Die Hyperleukocytose und die Polynucleose werden im Anfang von toxi-infektiösen Psychosen und bei Agitationszuständen angetroffen.

4. Unbedeutende Hyperleukocytose mit relativer Lymphocytose ist Zeichen einer schwachen Reaktion des Organismus gegen eine chronische Infektion.

5. Die Eosinophilie ist ein ,kritisches Zeichen".

Weichen demnach die Resultate dieser Autoren teilweise recht sehr voneinander ab, so gilt dies in noch bedeutend höherem Grade von den Serienuntersuchungen, die in der Literatur vorliegen.

Der erste, der eine größere hierhergehörige Untersuchung angestellt hat, ist Bruce, der teils allein, teils zusammen mit seinem Assistenten Peebles, eine ganze Reihe von Aufsätzen über Serienuntersuchungen bei verschiedenen Geisteskrankheiten veröffentlicht hat. Bei den wenigen Katatoniefällen, die er untersucht hat, hat er während des ersten akuten Stadiums eine mittelstarke Leukocytose gefunden, die vor allem die neutrophilen, polymorphkernigen Zellen betraf. Während die Temperatur sich erhöht, steigt dann diese Leukocytose, worauf der Patient, wenn das Fieber seinen Höhepunkt erreicht hat, in Stupor verfällt, wobei die Leukocytenanzahl rasch abnimmt und eine mäßige Hyperleukocytose, nun überwiegend Lymphocytose und Eosinophilie, bestehen bleibt, letzteres konstant in allen Fällen, die zur Heilung gelangen.

Bei Hebephrenie fand Bruce in der Regel Werte um 12 000-14000 herum mit Steigerungen dann und wann hinauf bis zu 30000 , vorzugsweise auf einer Vermehrung der großen mononucleären Zellen beruhend, wie in einigen Fällen 20-30\% der Gesamtanzahl Leukocyten ausmachten.

Zwei andere englische Forscher, Howard und Mc. Dowall, bestätigten im großen und ganzen Bruces Befunde. Mc. Dowall hat unter anderem einige Fälle von ,depression with exeitement" untersucht, die zum größten Teil zweifellos Dementia praecox-Fälle sind. $\mathrm{Er}$ findet stets Leukocytose $\mathrm{zu} 10000-20000$, vorzugsweise polymorphkernige. Gelangt der Fall zur Heilung, so nimmt die Leukocytose zuerst ab und steigt dann wieder; die Abnahme trifft vorzugsweise die polymorphkernigen Zellen. In Fällen, die nicht zur Heilung gelangen, variiert die Leukocytenanzahl sehr stark, der Prozentsatz polymorphkerniger Zellen ist aber stets hoch. Geht der Fall in Demenz über, so sinkt die Gesamtzahl der Leukocyten wie auch der Prozentsatz 
polymor phken niger Zollen, der bis a ui $50 \%$ heruntergehen kann, was ein schlechtes Zeichen ist. Bei ,depression with excitement" fand Mc. Dowall selten eosinophile /ollen, im Gegensatz zụ dem Verhältnis bei „,acute mania", wo stets Eosinophilie vorhanden ist, wenigstens bei Fällen, die zur Heilung gelangen. Nach Mc Dowalls Ansicht gestaltet sich die Prognose am besten bei hoher neutrophiler Leukocytose + Eosinophilie, beides Zeichen dafür, daß der Körper kräftig reagiert.

Lépine und Popoff untersuchten unter anderen 16 Fälle von Dementia praecox; mehrere Blutproben, jerloch nicht tägliche, wurden von jedem Patienten entnommen. Sie fanden der Regel nach eine herabgesetzte Anzahl roter Blutkörperchen, ferner in allen chronischen Fällen Lymphocytose. Die Anzahl der eosinophilen Zellen variierte ungeheuer bei verschiedenen Individuen und bei verschierlenen Gelegenheiten. In einigen Fällen beobachteten sie starke Oszillationen in der Jusammensetzung des Blutes als Vorläufer von Veränderungen im psychischen Zustande. Die neutrophile Polynucleose betrachten sie als Zeichen einer kräftigen Reaktion des Körpers und somit als prognostisch günstig, besondlers wenn sie mit Eosinophilie verbunden ist.

L undvall, der eine Menge sehr vollständiger Serienuntersuchungen angestellt hat, bei denen er die Patienten monatelang, zeitweise Tag für Tag, verfolgt hat, ist zu folgenden Resultaten gekommen.

Patienten, die an Dementia praecox leiden, weisen während Zeiten, wo sie von psychischen Reizungsphänomenen frei sind, keine anderen Blutveränderungen auf als eine niedrige Gesamtmenge weißer Blutkörperchen mit relativer Lymphocytose sowie scheinbarer Polycytämic, welch letztere Lundvall als auf peripherischen vasomotorischen Störungen beruhend auffaßt. Bei Patienten jedoch, deren Krankheitsverlauf zeitweise durch starke Reizungsphänomene, motorische und psychische Unruhe, unterbrochen wird, findet Lundvall periodenweise wiederkehrende typische Veränderungen, die er ,Blutkrisen“ nennt, und die in Oligocytämie und Hyperleukocytose mit prozentischer neutrophiler Polynucleose bestehen. Die eosinophilen Zellen zeigen zuerst eine Abnahme, auf dem Höhepunkt der Krise dagegen eine Zunahme, die noch nach Ende der Krise bestehen bleibt. Oft verlaufen diese ,Blutkrisen“ ohne andere sichtbare Symptome, sind sie aber hinreichend intensiv, so sind sie von motorischer und psychischer Unruhe begleitet. Lundvall faßt die Blutkrisen als Zeichen einer Toxinanhäufung im Blute auf.

Die Resultate dieser Untersuchungen sind, wie gesagt, ziemlich schwer in Utbereinstimmung miteinander zu bringen. Da weitere Untersuchungen somit vonnöten sind, und jeder genau untersuchte Fall von Bedeutung für die Klärung der Frage sein muß, so habe ich es, obwohl meine Untersuchung nur einen Fall betrifft, für gerechtfertigt erachtet, 
das Ergebnis derselben vorzulegen, um so mehr, als so vollständig, während längerer Zeit täglich angestellte Blutuntersuchungen zuror nur von Lundvall veröffentlicht worden sind und außerdem der Fall an und für sich wegen seines Verlaufes von Interesse ist.

Die Blutproben wurden stets so entnommen, daB die Verdauungsleukocytose vermieden wurde, in der Regel einmal täglich, gleich vor der Mittagsmahlzeit. An den Tagen, wo der Zustand des Patienten aus dem ruhigen in den unruhigen überging, und am Tage gleich clanach entnahm ich zwei oder drei Proben.

Die Proben wurden aus der Fingerkuppe unter Beobachtung der gewöhnlichen Vorsichtsmaßregeln entnommen. Ich bediente mich des Thomas - Zeißschen Apparates zum Zählen sowohl der roten als der weißen Blutkörperchen. Bei den Zählungen verfuhr ich in der Weise, daß ich zuerst 5-6 Tropfen ausbließ und danach einen Tropfen in die Zählkammer unter Achtgabe darauf brachte, daß die Newtonschen Farbenringe stets zu sehen waren als ein Kriterium dafür, daß keino Flüssigkeit zwischen dem Deckglase und dem äußeren, erhöhten Rande der Zählkammer vorhanden war. Bei der Bestimmung der Erythrocytenanzahl zählte ich mindestens 200 kleine Felder, gewöhnlich bei 2 oder 3 verschiedenen Tropfen. Bei der Bestimmung der weißen Blutkörperchen zählte ich mindestens 7 Tropfen, d. h. 2800 Quadrate, durch. Zur Differentialzählung der verschiedenen Arten weißer Blutkörperchen benutzte ich Trockenpräparate, färbte mit May-Grünwalds Farbe, zählte mittels verschiebbaren Objekttisches 400 weißo Blutkörperchen durch und berechnete daraus das prozentische Verhältnis zwischen den verschiedenen Arten.

In Tabelle I habe ich die gefundenen Werte zusammengestellt; es sind clabei für jede Untersuchung angegeben die Gesamtzahl weißer Blutkörperchen, der Prozentsatz neutrophiler Leukocyten, eosinophiler Leukocyten, Lymphocyten und Úbergangszellen, sowie die Gesamtzahl neutrophiler Leukocyten und Lymphocyten. Während der Zeit vom 5. IV. bis 30. IV. betrifft die Cntersuchung auch die Anzah1 roter Blutkörperchen. In der letzten Spalte habe ich mit einigen Worten den psychischen Zustand für jeden Tag angegeben.

In Tabelle II habe ich graphisch die Variationen in der Gesamtanzahl roter und weißer Blutkörperchen sowie in lem Prozentsatz neutrophiler Leukocyten, Lymphocyten und eosinophiler Zellen dargestellt, und Tabelle III zeigt die Gesamtanzahl neutrophiler Leukocyten und Lymphocyten für jede Untersuchung.

Betrachten wir diese Tabellen und Kurven, so fällt sofort ein Wechsel der Gesamtanzahl weißer Blutkörperchen in die Augen, ein Wechsel der in einer deutlichen, ziemlich regelmäßigen Wellenbewegung verläuft, wobei die Wellentäler in die freien Intervalle und die Wellenberge in die 
Tabelle I.

\begin{tabular}{|c|c|c|c|c|c|c|c|c|c|}
\hline 音 & 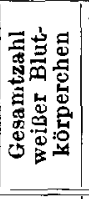 & 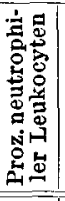 & 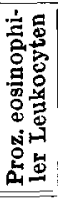 & 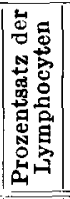 & 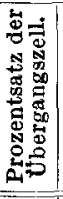 & 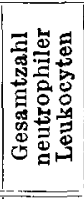 & 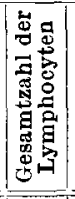 & 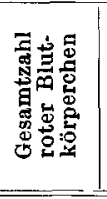 & Anmerkungen \\
\hline 11. 3 . & 5100 & 58 & 1 & 37 & 4 & 2960 & 1890 & - & $\begin{array}{l}\text { Morgens in die ruhige Abteilung } \\
\text { verbracht. }\end{array}$ \\
\hline 12. & 5000 & 69 & 2 & 25 & 4 & 3450 & 1250 & - & $\begin{array}{l}\text { Auf Besuch in der Stadt, sym- } \\
\text { ptomenfrei. }\end{array}$ \\
\hline 13. & 4600 & 51 & 1 & 44 & 4 & 2350 & 2050 & 一 & do. \\
\hline 14. & 6200 & 65 & 1 & 31 & 3 & 4030 & 1920 & 一 & Symptomenfrei. \\
\hline 15. & 6400 & 68 & 1,25 & 26 & 5 & 4350 & 1670 & - & do. \\
\hline 16. & 6700 & 62 & 1 & 31 & 6 & 4150 & 2110 & $\longrightarrow$ & do. \\
\hline 17. & 7100 & 68 & 0,5 & 28,5 & 3 & 4830 & 2020 & 一 & $\begin{array}{l}\text { Spricht stoBwe } \\
\text { eine „Starrk }\end{array}$ \\
\hline $1 \mathrm{~nm}$. & 8200 & 65 & 0,5 & 30,5 & 4 & 5330 & 2500 & 一 & $\begin{array}{l}\text { Beginnt unruhig zu } \\
\text { alle ,du“, widers } \\
\text { Athetosebewegun }\end{array}$ \\
\hline 18. $\{5 \mathrm{~nm}$. & 8800 & 58 & 1,5 & 39 & 1,5 & 5100 & 3430 & - & $\begin{array}{l}\text { Wird um } 5 \text { Uhr in die unruhige } \\
\text { Abteilung verbracht. }\end{array}$ \\
\hline $6 \mathrm{~nm}$. & 11000 & 45 & 1 & 48 & 6 & 4950 & 5300 & 一 & $\begin{array}{l}\text { Manisch, macht Scherze; mäßige } \\
\text { motorische Unruhe. }\end{array}$ \\
\hline $10 \mathrm{vm}$. & 11300 & 61 & 1 & 35 & 3 & 6890 & 3960 & - & $\begin{array}{l}\text { Starke motorische Unruhe, ab- } \\
\text { wechselnd gehobene und de- } \\
\text { primierte Stimmuug. }\end{array}$ \\
\hline $2 \mathrm{~nm}$. & 10600 & 61 & 1 & 37 & 1 & 6470 & 3980 & 一 & do. \\
\hline $6 \mathrm{~nm}$ & 8600 & 54 & 1 & 43 & 2 & 4730 & 2700 & - & do. \\
\hline $10 \mathrm{vm}$. & 9400 & 68 & 0,5 & 29,5 & 2 & 6390 & 2820 & - & Ängstlich. \\
\hline $2 \mathrm{~nm}$. & 11300 & 66 & 0 & 30 & 4 & 7460 & 3390 & - & \\
\hline 21. & 7400 & 62 & 1 & 37 & 1 & 4600 & 2740 & - & Sehr agitiert, aufgeräumt. \\
\hline 22. & 9200 & 65 & 0,5 & 32 & 2,5 & 5980 & 2940 & - & te und de- \\
\hline 23. & 6300 & 65 & 1,5 & 32 & 1,5 & 4090 & 2020 & - & $\begin{array}{l}\text { Rubig, geordnet und klar, ein } \\
\text { wenig gedämpft und deprimiert. } \\
\text { In die ruhige Abteilung verbr. }\end{array}$ \\
\hline 24 & 5700 & 60 & 1 & 37 & 2 & 3420 & 2330 & - & Besonnen und klar. \\
\hline 25. & 5100 & 59 & 1 & 39 & 1 & 3110 & 1990 & - & Symptomenfrei. \\
\hline 26. & 5800 & 59 & 2 & 38 & 1 & 3420 & 2200 & 一 & do. \\
\hline 27. & 5300 & 60 & 0,5 & 34,5 & 5 & 3180 & 1860 & - & do. \\
\hline 28. & 6300 & 58 & 0,5 & 40,5 & 1 & 3650 & 2520 & - & do. \\
\hline 29 . & 6400 & 58 & 1 & 34 & 7 & 3710 & $2180 \mid$ & - & do. \\
\hline 30. & 6100 & 57 & 2 & 40 & 1 & 3480 & 2440 & - & Nicht ganz gut. \\
\hline 31. $\{2 \mathrm{~nm}$. & 7800 & 57 & 2 & 37 & 4 & 4450 & 2880 & - & $\begin{array}{l}\text { Aufgeräumt, nennt alle „du“, un- } \\
\text { ruhig. }\end{array}$ \\
\hline $6 \mathrm{~nm}$. & 10600 & 53 & 0,5 & 45 & 1,5 & 5620 & 4770 & 一 & $\begin{array}{l}\text { Wird um } 3 \text { Uhr in die unruhige } \\
\text { Abteilung verbracht, manisch. }\end{array}$ \\
\hline $2 \mathrm{~nm}$. & 9800 & 60 & 1 & 36 & 3 & 6080 & 3530 & - & Niedergedrückt, agitiert. \\
\hline$\cdot\{7 \mathrm{~nm}$. & 9500 & 62,5 & 0,5 & 36 & 1 & 5990 & 3420 & - & do. \\
\hline $2 \mathrm{~nm}$. & 12200 & 63,5 & 0,5 & 33 & 3 & 7690 & 4030 & - & Liegt starr, jammert. \\
\hline$\{6 \mathrm{im}$. & 12000 & 62 & 0,5 & 36 & 1,5 & 7440 & 4320 & - & do. \\
\hline 3. & 12600 & 60 & 0,5 & $36, \overline{0}$ & $\mathbf{3}$ & 7560 & 4660 & - & Manisch, sehr agitiert. \\
\hline 4. & 9000 & 53 & 1 & 44 & 2 & 4730 & 3960 & - & Wird nur mit Mühe gehalten. \\
\hline 5. & 9800 & 55 & 3 & 39 & 3 & 5490 & 3820 & 4800000 & $\begin{array}{l}\text { Morgens agitiert, wurde später } \\
\text { ruhig. Um } 2 \text { Uhr in die ruhige } \\
\text { Abteilung verbracht. }\end{array}$ \\
\hline 6. & 7200 & 57,5 & 1,5 & 37 & 4 & 4180 & 2660 & 5200000 & $\begin{array}{l}\text { Symptomenfrei, auf Besuch in der } \\
\text { Stadt. }\end{array}$ \\
\hline 7. & 6600 & 55 & $\mathbf{2}$ & 37 & 6 & 3630 & 2440 & 4600000 & Symptomenfrei. \\
\hline 8. & 7900 & 60 & 1 & 37 & 2 & 4740 & $|2880|$ & $|5450000|$ & do. \\
\hline
\end{tabular}


Fortsetzung von Tabelle I.

\begin{tabular}{|c|c|c|c|c|c|c|c|c|c|}
\hline 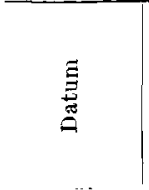 & 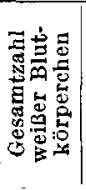 & 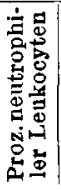 & 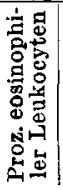 & 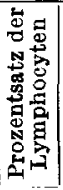 & 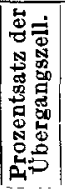 & 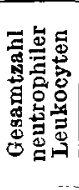 & 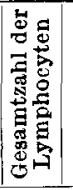 & 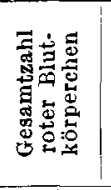 & Anmerkungen \\
\hline 9. 4 . & 8800 & 59 & 1 & 37 & 3 & 5190 & 3260 & 5840000 & Symptomenfrei. \\
\hline 10. & 9200 & 55,5 & 1,5 & 34 & 9 & 5150 & 3130 & 4100000 & do. \\
\hline 11. & 7300 & 60 & 0,5 & 32 & 7,5 & 4880 & 2340 & 4200000 & Schlechter. \\
\hline $2 \mathrm{~nm}$ & 9000 & 61 & 1 & 31 & 4 & 5760 & 2790 & 4200000 & $\begin{array}{l}\text { Um } 12 \text { Uhr in die unruhige } \mathrm{Ab} \text { - } \\
\text { teilung verbracht, manisch, un- } \\
\text { ruhig. }\end{array}$ \\
\hline $16 \mathrm{~nm}$. & 10600 & 65 & 1 & 30 & 4 & 6890 & 3180 & 4500000 & Motorische Unruhe. \\
\hline 13. & 11000 & 68 & 0,5 & 26,5 & 5 & 7480 & 2970 & 4640000 & Sehr starr, weint ab und zu. \\
\hline 14. & 11300 & 71 & 0,5 & 25 & 3,5 & 8020 & 2820 & 5160000 & do., spricht nichts. \\
\hline 15. & 9400 & 61 & 1 & 35 & 3 & 5730 & 3290 & 5800000 & $\begin{array}{l}\text { Aufgeräumt, sehr motorisch agi- } \\
\text { tiert. }\end{array}$ \\
\hline 16. & 9000 & 68 & 0,5 & 26 & 5,5 & 6160 & 2290 & 6100000 & $\begin{array}{l}\text { Sehr manisch, macht Witze, muls } \\
\text { durch } 2 \text { wächter gehalten werd. }\end{array}$ \\
\hline 17. & 7000 & 62 & 1 & 34 & 3 & 4330 & 2380 & 5600000 & $\begin{array}{l}\text { Ruhig, wird morgens in die ruhi- } \\
\text { ge Abteilung verbracht. }\end{array}$ \\
\hline 18. & 7200 & 57 & 1 & 40 & 2 & 4070 & 2950 & 5700000 & Ruhig, in der stadt. \\
\hline 19. & 6200 & 58 & 0,5 & 39 & 2,5 & 3600 & 2420 & 5300000 & do. \\
\hline 20. & 6300 & 52 & 1,5 & 46 & 0,5 & 3280 & $2900 \mid$ & $5300000 \mid$ & do. \\
\hline 21. & 6800 & 58 & 2 & 37 & 3 & 3940 & 2520 & 5600000 & do. \\
\hline 22. & 5800 & 58 & 1,5 & 38 & 2,5 & 3360 & 2200 & 5300000 & do. \\
\hline 23. & 5000 & 55 & 1 & 42 & 2 & 3250 & 2480 & 5760000 & do. \\
\hline $11 \mathrm{vm}$. & 8000 & 60 & 0,5 & 38,5 & 1 & 4800 & 3080 & 5360000 & $\begin{array}{l}\text { In der Nacht unruhig, wird um } \\
\text { lo Uhr morgens in die unruhige } \\
\text { Abteilung verbracht, nicht agi- } \\
\text { tiert. }\end{array}$ \\
\hline ( 2 иाı. & 9700 & 50 & 2,5 & 45 & 2,5 & 4850 & 4370 & 5200000 & $\begin{array}{l}\text { Macht Witze, keine motorische } \\
\text { Unruhe. }\end{array}$ \\
\hline 25. & 7900 & 56 & 1 & 42 & 1 & 4500 & 3240 & 4800000 & $\begin{array}{l}\text { Sitzt zusammengerollt, jammert, } \\
\text { sehr cyanotisch, spriclit nichts. }\end{array}$ \\
\hline 26 & 9800 & 76 & 0,5 & $\underline{93}$ & 0,5 & 7450 & 2250 & 46850000 & do., nicht so ängstlich. \\
\hline 27 & 9000 & 65 & 0,5 & 34 & 0,5 & $5 \$ 80$ & 3060 & 5160000 & $\begin{array}{l}\text { Manisch, sehr agitiert, wird mit } \\
\text { Mühe von } 2 \text { Wärten gelialten, } \\
\text { macht witze. }\end{array}$ \\
\hline 28. & 9400 & 60 & 0,5 & 34 & 5,5 & 5640 & 3200 & 5400000 & do. \\
\hline 29 & 7600 & 54 & 1 & 42 & 3 & 4100 & 3190 & 5560000 & $\begin{array}{l}\text { Immer unruhig, Stimmen wech- } \\
\text { selnd. }\end{array}$ \\
\hline 30. & 5700 & 52 & 3 & 43 & 2 & 2960 & 2450 & 5400000 & $\begin{array}{l}\text { Morgens in die rubige Abteilung } \\
\text { verbracht, symptomenfrei. }\end{array}$ \\
\hline
\end{tabular}

Unruheperioden fallen. Während der freien Intervalle hat Patient eine Gesamtanzahl weißer Blutkörperchen von 5000-8000, d. h. eine völlig normale Ziffer; während der Unruheperioden steigt die Anzahl weißer Blutkörperchen auf 9000-12 000, eine zwar recht unbedeutende, aber bei allen den vier untersuchten Unruheperioden völlig deutlich hervortretende und konstante Zunahme.

Prüfen wir diese Zunahme näher, so zeigt es sich, daß sie ziemlich gleichmäßig auf alle Arten weißer Blutkörperchen verteilt ist, doch zeigen die neutrophilen polymorphkernigen Leukocyten eine etwas stärkere Zunahme als die übrigen, besonders in den zwei letzten Unruhe- 


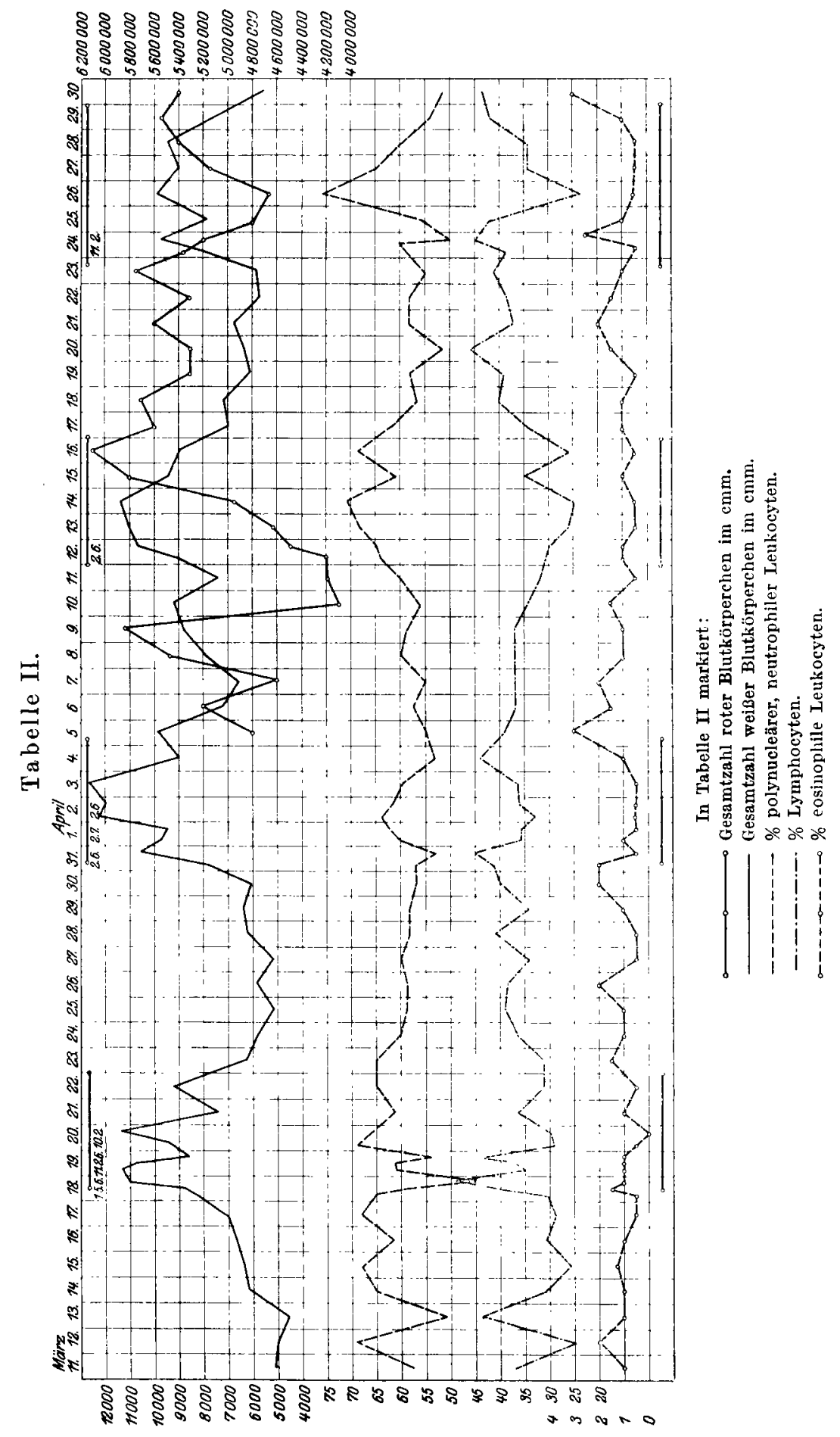


perioden, wo die Steigerung der Gesamtanzahl ganz deutlich der Hauptsache nach auf einer Steigerung der Anzahl der Neutrophilen beruht.

Während der freien Intervalle hält sich der Prozentsatz neutrophiler Leukocyten zwischen 45 und $65 \%$, gewöhnlich um $55 \%$ herum, der Prozentsatz Lymphocyten dagegen zwischen 30 und $45 \%, \mathrm{~d}$. h. es liegt eine relative Lymphocytose vor. Während der Unruheperioden steigt, wie gesagt, die Anzahl neutrophiler Zellen etwas mehr als die Anzahl Lymphocyten, so daß die relative Lymphocytose abnimmt und das prozentische Verhältnis zwischen neutrophilen Leukocyten und Lymphocyten sich mehr dem normalen nähert, ohne es jedoch zu erreichen.

Tabelle III, in der ich für jeden Tag die Gesamtanzahl teils neutrophiler polymorphkerniger Leukocyten, teils der Lymphocyten ausgerechnet habe, zeigt die interessante Tatsache, daß die Lymphocyten stets die größte Zunahme am ersten Tage der Unruheperiode aufweisen, um dann rasch zurückzugehen, während die Anzahl der neutrophilen Leukocyten bis zur Mitte der Unruheperiode ansteigt, wo sie am größten ist, um danach so allmählich, wie sie zugenommen hat, auch wieder abzunehmen.

Eine Eosinophilie, wie mehrere Autoren sie bei Dementia praecox gefunden haben, habe ich nicht konstatieren können; eher das

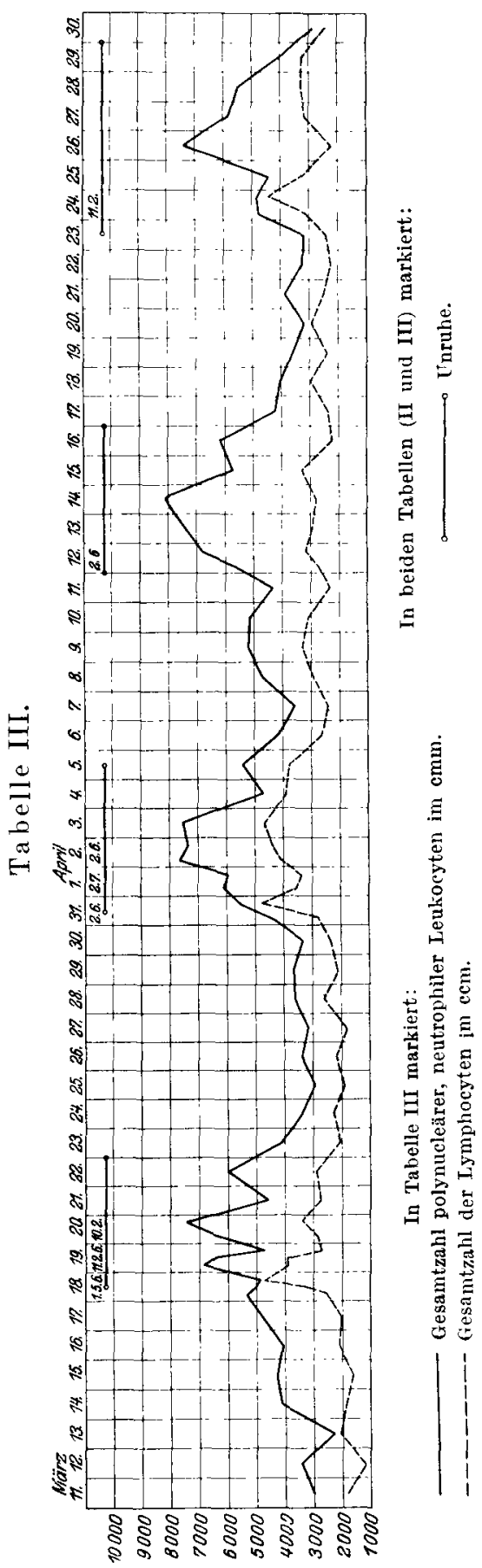


Gegenteil, indem kein einziges Mal der Prozentsatz eosinophiler Zellen die Ziffer 3 überschritten hat. Die eosinophilen Zellen zeigen auch nicht solche Schwankungen der Anzahl wie die übrigen weißen Blutkörperchen.

Die „Ubergangszellen" zeigen gleichfalls kein bemerkenswertes Verhalten.

Die Kurve der roten Blutkörperchen ist sehr unregelmäßig. Sie weist am 10. April, gegen das Ende eines freien Intervalls, einen heftigen Fall von 5400000 auf 4100000 auf, steigt dann während der Unruheperiode an und erreicht zu Ende derselben die Ziffer 6100000 . Während des nächsten freien Intervalls halten sich die Erythrocyten um die Ziffer 5500000 herum und fallen dann mitten während der darauffolgenden Unruheperiode auf den Wert 4650000 hinab.

Wie aus dem Bericht über die Literatur hervorging, hat man im allgemeinen als typisches Blutbild bei Dementia praecox Hyperleukocytose mit relativer Lymphocytose und Eosinophilie angegeben. Mein Fall zeigt keine stärker hervortretende Hyperleukocytose, auch keine Eosinophilie, wohl aber eine deutliche relative Lymphocytose. Ferner zeigt mein Fall schöne „Blutkrisen“, ähnlich den von Lundvall beschriebenen. Er fand bei diesen Krisen Oligocytämie, Hyperleukocytose mit prozentischer neutrophiler Polynucleose sowie eine Eosinophilie während des späteren Teils der Krise. Auch ich finde eine, allerdings mäßige, Hyperleukocytose, d.h. eine Zunahme der Gesamtanzahl weißer Blutkörperchen mit einer im allgemeinen unbedeutenden prozentischen Zunahme der neutrophilen Zellen (oder Abnahme der relativen Lymphocytose, wenn man so will). Mein Fall zeigt auch eine Andeutung von Oligocytämie während der Krisen; dagegen habe ich nicht die Eosinophilie konstatieren können, die Lundvall beschreibt.

Außer Lundvall hat Pförtner ähnliche Blutkrisen bei ein paar Fällen von Dementia praecox beobachtet, und auch Lépine und Popoff berichten von Oszillationen im Blutbilde als Vorläufern von Veränderungen im Zustande des Pạtienten, ohne sie jedoch näher zu beschreiben. Daß nicht mehr Autoren diese Blutkrise gefunden haben, beruht wohl vermutlich darauf, daß sie nicht bei allen Fällen von Dementia praecox vorhanden sind, sondern nur bei denen mit periodischem Verlauf, teilweise beruht es wohl aber darauf auch, daß so wenige Autoren Serienuntersuchungen angestellt haben, und ohne solche können die Krisen natürlich nicht hervorteten. Wenn daher H. Krueger sagt: „Der Theorie Lundvalls, das sei gleich hervorgehoben, ist niemand gefolgt, seine Angaben sind nirgends bestätigt", so liegt wohl die Erklärung, wenigstens für den letzteren Umstand, darin, daß die meisten, wie Krueger selbst, nur eine einzige Probe von jedem Patienten genommen haben.

Die meisten Autoren, die Blutuntersuchungen an Dementia-praecox- 
Kranken angestellt haben, haben aus dem Blutbilde Schlüsse auf die Ätiologie der Krankheit zu ziehen versucht. Im allgemeinen ist man dabei der Ansicht gewesen, daß das Blutbild mit ziemlich großer Wahrscheinlichkeit für eine infektiöse oder toxische Ursache der Krankheit spräche, eine Ansicht, die mit recht großer Einigkeit von Autoren vertreten wird, die voneinander beträchtlich abweichende Blutbilder gefunden haben. Dieser letztere Umstand erklärt sich daraus, daß das Blutbild teils bei verschiedenen Infektionen und teils bei verschiedenen Phasen derselben Infektion beträchtlich verschieden ist. In letzter Zeit hat man auf die Ähnlichkeit des Blutbildes bei Dementia praecox und bei einigen auf Störungen der inneren Sekretion beruhenden Krankheitszuständen, wie Morbus Basedowii, Myxödem, und auch bei Krankheiten der Hypophyse, der Nebennieren und beim Status thymolymphaticus hingewiesen. Bei diesen Zuständen hat man im allgemeinen relative Lymphocytose und bisweilen Eosinophilie gefunden, d. h. ein Blutbild ähnlich dem, das jedenfalls das gewöhnlichste bei Dementia praecox zu sein scheint, ein Umstand, der dafür spricht, daß Störungen der inneren Sekretion bei Dementia praecox vorhanden sind, der aber natürlich keine Auskunft darüber liefert, wo die primäre Krankheitsursache liegt. Ob die Störungen der inneren Sekretion, die Funktion des Gehirns oder des Blutes das Primäre ist, oder ob sie vielmehr alle drei die Folge einer gemeinsamen Ursache sind, die wir noch nicht kennen, das läßt sich an der Hand des Blutbildes nicht weiter entscheiden. In allerletzter Zeit hat man ja auf serologischem Wege versucht, der Lösung dieser Frage näher zu kommen.

Was die „Blutkrisen", jene Wellenbewegung im Blutbilde, die mein Fall zeigt, betrifft, so wäre es natürlich denkbar, daß sie ganz einfach eine Folge der verschiedenen Weise des Patienten wären, sich während der freien Intervalle und der Unruheperioden zu verhalten, $\mathbf{d}$. h. daß die Zunahme der Gesamtzahl weißer Blutkörperchen, die während der Unruheperioden stattfindet, auf der vermehrten Muskelarbeit beruhte, die der Patient dann leistet, eventuell auf einer veränderten Konzentration des Blutes. Man weiß aus Untersuchungen von Grawitz u. a., daß Muskelarbeit von einer bedeutenden Zunahme der Anzahl der weißen Blutkörperchen begleitet ist. Grawitz fand, daß diese Zunahme sehr rasch eintrat und am größten während der ersten 10-20 Minuten war. Die Zunahme war sehr erheblich, so daß im allgemeinen die Anzahl der weißen Blutkörperchen auf das Drei- oder Vierfache ihrer Anzahl während der Ruhe hinaufsprang. Während der ersten und bisweilen auch während der zweiten halben Stunde bestand eine ausgesprochene Lymphocytose, die dann in polynucleare Leukocytose überging. Daß diese Vermehrung der weißen Blutkörperchen nicht nur auf einer veränderten Verteilung des Blutes beruht, zeigte Grawitz dadurch, daß 
er Proben teils aus einer Vene, teils aus den Capillaren entnahm, wobei das Blut genau die gleiche Zusammensetzung zeigte.

Lundvall weist bestimmt den Gedanken daran ab, daß die Muskelarbeit allein die Ursache dieser Blutkrisen wäre, indem er darauf hinweist, daß einerseits, wie die Erfahrung bei den Manien zeigt, solchenfalls gleichzeitig mit der Leukocytose eine Erythrocytose vorhanden sein müßte, beide Zeichen einer vermehrten molekularen Konzentration des Blutes, während bei diesen Blutkrisen im Gegenteil Oligocytämie herrscht, und daß andererseits die Blutkrisen bisweilen mehrere Tiuge vor der psychischen und physischen Unruhe beginnen, ja, sogar sozusagen abortiv verlaufen können, d.h. ohne daß eine Veränderung des psychischen Status eintritt.

In meinem Falle zeigt die Anzahl der Erythrocyten kein konstantes Verhalten, und wenn man die Kurven und die Tabelle betrachtet, so muß man sich sagen, daß nirgends deutliche Blutveränderungen v or dem Einsetzen des Unruhezustandes hervortreten. Es ist jedoch auffällig, daß weder der Beginn noch der Rückgang der Leukocytose sich ebenso rasch vollzieht wie der Beginn und Rückgang der psychischen und physischen Unruhesymptome und offenbar langsamer, als wie man es auf Grund der Grawitzschen Untersuchungen bei einer allein auf Muskelarbeit beruhenden Leukocytose erwarten sollte, und daß auch dabei bei weitem nicht die Höhe erreicht wird, wie sie cine Arbeitsleukocytose nach Grawitz aufzuweisen pflegt; ferner auch, daß die Leukocytose stets am höchsten mitten in der Unruheperiode ist, wo der Patient stets deprimiert ist und wenigstens scheinbar bedeutend weniger Muskelarbeit leistet als zu Beginn und am Schlusse derselben. Möglich ist ja indessen, daß die intensive Spannung, in der sein ganzer Körper sich wïhrend dieser Tage der Depression befindet, mehr Muskelarbeit bedeutet als die starke motorische Agitation zu Beginn und am Schlusse der Anfälle.

Das Wahrscheinlichste scheint mir jedenfalls zu sein, daß die Muskelarbeit ein wichtiger und vielleicht der wichtigste Faktor für die Entstehung dieser Blutkrisen ist, daß er aber nicht den einzigen darstellt, und das Resultat meiner Untersuchung ist durehaus nicht unvereinbar mit Lundvalls Theorie, daß die Blutkrisen Zeichen einer Ansammlung für Dementia praecox spezifischer Toxine im Blute sind, eher scheint es mir eine gewisse Stütze für diese Theorie zu liefern.

Die leichte Temperatursteigerung, die sich, besonders im Anfange, in den Unruheperioden findet, beruht wohl dagegen zweifellos auf der starken Muskelarbeit, da eine solche erfahrungsgemäß eine rasch vorübergehende Temperatursteigerung bei gesunden Personen hervorruft. Eine Einwirkung auf das Blutbild kann diese Temperatursteigerung an und für sich mit aller Sicherheit nicht haken. Man weiß nämlich durch 
Untersuchungen unter anderem von Rovighi, daß Tiere, deren Körpertemperatur durch Aufenthalt im Thermostat bis zu $3^{\circ}$ über die normale hinaus erhöht worden war, keine Vermehrung, sondern im Gegenteil eine Verminderung der Leukocytenanzahl aufwiesen, und Naegeli sagt in seinem Buch über "Blutkrankheiten und Blutdiagnostik", da B.3 die Leukocytose bei Infektionskrankheiten ausschließlich auf den Toxinen beruht, wobei er hinzufügt: „Daß das Fieber selbst keine Leukocytose hervorruft, ist klar und bedarf keiner weiteren Erörterung".

Was schließlich die Erklärung des obenerwähnten, in Tabelle III deutlich hervortretenden Lmstandes betrifft, daß die Lymphocyten stets clie größte Zunahme am ersten Tage der Unruheperiode zeigen, um dann zurückzugchen, während die neutrophilen Leukocyten bis zur Mitte der Periode, wo ihre Anzahl am größten ist, zunehmen, um dann erst zurückzugehen, so könnte sie vielleicht darin erblickt werden, daß3 die in Lymphdrüsen und Milz befindlichen Lymphocyten leichter durch die heftige Muskelarbeit in den Kreislauf eingepumpt werden, als die Leukocyten. Daß die Lymphocyten auf normale Menge zurückgehen, während die Leukocytenanzahl steigt, könnte möglicherweise eine Stïtze für die von vielen gemachte Annahme abgeben, daß Lympho"yten in Leukocyten übergehen.

Zum Schluls ist es mir eine angenehme Pflicht, meinem hochverehrten früheren (hef, Herm Professor Dr. Gadelius, meinen Dank für das grolie Entgegenkommen, mit dem er mir den Fall äberfassen hat, und für das wohlwollende Interesse, das er meiner Arbeit entgegengebracht hat, anszusprechen.

\section{Literaturverzeichnis.}

1. Bruee, L., Bacteriological and elinical ohservations of eases suffering from acut continous mania. Journ. Ment. Sc. 1913.

$2,-$ - Further clinical observations in cases of acute mania, particularly adnlescent mania. Ibidem 1903.

3. - and Perbles, A. U. S., Clinical and experimental observations on kattonia, Ibidem 1903.

4. - - - Quantitative and qualitative leucocyt counts in various forms of mental disease. Ibidem 1904.

5. - The symptoms and etiologi of mania. Ibidem 1908.

6. I)idr, M., Etude cytologique, hactériologique et expérimentale du sang chez les aliénés. XVI. Congr. des méd. aliénistes et nourologistes. Lille 1906.

7. - et Chénais, L., Recherches urologiques et hématologiques dans la démence précoce. Annales méd. psych. 60, 1902.

8. M. Dowall, Colin, Leucocytosis: Its relation to and significans in acute mental disorders. Journ. of mental Se. 1908.

9. - The Lencocyte and the acute insanities. Ibidem 1909.

10. Grawitz, Üher myogene Lencocytosen. Weutsche med. Wochenschr. 1910, Nr. 29. 
500 G. Kahlmeter: Blutuntersuchungen bei einem Fall von Dementia praecox.

11. Heilemann, Blutuntersuchungen bei Dementia praecox. Allgem. Zeitschr. f. Psych. 1910.

12. Howard Carlisle, The systematic estimation of the leucocytosis in certain cases of insanity; with special reference to the totaemie theory. Journ. mental Sc. 1910.

13. Itten, Heilversuche mit Nucleininjektionen bei Schizophrenie (Dementia praecox). Mit einer vorläufigen Mitteilung über cytologische Blutbefunde bei dieser Krankheit. Zeitschr. f. ges. Psych. u. Neur. ', 1911.

14. Kuhn, William, Toxaemia of insanity. Journ. of the Amer. med. Assoc. 50, Nr. 15.

15. Krueger, H., Über die Cytologie des Blutes bei Dementia praecox. Zeitsehr. f. d. ges. Neur. u. Psych. 14. 1913.

16. Lépine, J., und Popoff', V. S., Recherches sur les variations cytologiques du sang chez les aliénés. L'encéphale 1908.

17. Lu ndvall, H., Blodundersökningar på sinnessjuka. Hygiea 1907.

18. - Über Blutveränderungen bei Dementia praecox nebst einem Versuche einer Art spec. Terapi. Tidskrift for Nordisk retsmedicin og psykiatri 10. 4. 1912.

19. Naegeli, Blutkrankheiten und Blutdiagnostik. Leipzig 1912.

20. Pförtner, O., Die weißen Blutkörperchen beim Jugendirresein. Archiv f. Psych. u. Nervenheilk. 50, Heft 2, 1912.

21. Purdrum u. Wells, Dementia praecox. Journ. of the Amer. med. Assoc. 51, Nr. 1 .

22. Rougeau, R., Contribution à l'étude du sang chez les aliénés. Thèse, Tou. louse 1906.

23. Rovighi, Zit. Naegeli.

24. Sandri, La formola emo-leucocytaria nelle demenza pracoce. Rivista di Patologica nervosa e mentale 1905. 